\title{
A dispositio típica das cartas paulinas: tese e probatio segundo o ensinamento de Aristóteles*
}

\author{
[The pauline letters typical dispositio: thesis and \\ probatio, according to Aristotle's teaching]
}

\author{
Scott Normand Brodeur, S.J. ${ }^{*}$
}

\section{Resumo}

O presente artigo examina a exposição típica das cartas de Paulo. Defende-se que esta dispositio é uma herança do mundo cultural helênico, sobretudo de alguns conceitos fundamentais, contidos na Retórica de Aristóteles. Para isso, o texto está dividido em três momentos. No primeiro momento, tenta-se compreender melhor a riqueza cultural do Apóstolo das gentes, considera-se os seus três mundos. No segundo momento, examinamos a exposição típica das Cartas Paulinas: tese e probatio, segundo o ensinamento de Aristóteles. $\mathrm{O}$ artigo conclui com uma breve consideração da Carta aos Romanos, como exemplo típico da dispositio aristotélica.

Palavras-chave: Retórica; Paulo; Aristóteles; Helenismo.

\begin{abstract}
This article examines Paul's letters typical exposition. It defendes that this dispositio is a hellenic cultural world heritage, overall regarding to some fundamental concepts found up in Aristotle's Rethoric. The text is divided in three moments. In the first one, one tries understanding better the "gentium" Apostle's cultural richness, one considers his three worlds. In the second moment, one examines the paulines letters typical exposition: thesis and probatio, according to Aristotle's teaching. The article concludes with a short consideration concerning the letter to the Romans, as the aristotelian dispositio typical example.
\end{abstract}

Key-words: Rethoric; Paul; Aristotle; Helenism.

\section{Introdução}

Desejo agradecer ao coordenador do curso de Filosofia da

\footnotetext{
* Texto apresentado numa conferência dada no auditório G2 da Universidade Católica de Pernambuco, em agosto de 2015.

* Doutor em teologia. Professor de Novo Testamento no departamento de teologia da Universidade Gregoriana.
} 
vossa bela universidade pelo seu cordial convite feito a mim, para proferir esta conferência hoje. Não obstante o fato de que eu tenha concluído os meus estudos de filosofia há mais de trinta anos no Centre Sèvres de Paris, (a mesma faculdade da Companhia de Jesus onde estudou o vosso reitor, Pe. Pedro Rubens), para mim, é sempre uma alegria retomar em mãos um texto clássico como a Retórica de Aristóteles. A minha exposição está dividida em três momentos - o primeiro indício de que se segue uma abordagem antiga e clássica!

No primeiro momento, para compreender melhor a riqueza cultural do Apóstolo das gentes, consideramos os seus três mundos.

No segundo momento, examinamos a exposição típica das Cartas Paulinas: tese e probatio, segundo o ensinamento de Aristóteles. Com o auxílio de uma brevíssima citação da Retórica de Aristóteles como texto base, estaremos em condições de apreciar melhor a contribuição insubstituível do helenismo, na difusão do Evangelho de Deus no primeiro século depois de Cristo.

Enfim, chegaremos ao terceiro ponto: uma breve consideração da Carta aos Romanos como exemplo típico da dispositio aristotélica. Iniciemos o nosso estudo com uma consideração da formação intelectual e da identidade cultural de Paulo de Tarso.

\section{O Apóstolo Paulo, homem de três culturas}

Paulo foi definido por a A. Deissmann "um cosmopolita". $\mathrm{Na}$ realidade, na sua pessoa e na sua obra, se entrecruzam três mundos e três culturas: 1) Hebreu, por nascimento e religião ele 2) se exprime na língua e nas formas do helenismo; 3) é um cidadão romano que se enquadra lealmente no quadro do império.

R. Wallace e W. Williams ${ }^{1}$ preferem falar de três níveis culturais fundamentais. Segundo eles, uma cultura não exclui a outra, e Paulo participa das três ao mesmo tempo. A identidade do Apóstolo, é o resultado de uma construção complexa, baseada

\footnotetext{
${ }^{1}$ Per un'ottima presentazione di queste tre dimensioni dell'Apostolo, vedi R. Wallace - W. Williams, The Three Worlds of Paul of Tarsus, London 1998; C.J. ROETZEL, The World that Shaped the NT, Louisville 1985, 2002²; F. BIANCHINI, "Alla ricerca dell'identità dell'apostolo Paolo", 45-53.

150 - Universidade Católica dePernambuco
} 
sobre a sua cultura nativa, alimentada da multiforme cultura helenística que dominava todo o Oriente e da cultura emanada e difusa pelo poder dominante: Roma. Paulo era hebreu, helenista e romano.

\subsection{O hebraísmo}

O hebraísmo assinala-o indelevelmente desde o nascimento 2: "Sou um hebreu de Tarso da Cilícia" (At 21,39), declara ao tribuno romano que lhe pergunta no momento da sua prisão rumo à Jerusalém, indicando assim, pertencer à diáspora hebraica dispersa no mundo helenizado.

Diante dos detratores de Corinto, que lhe contestam a autoridade apostólica, ele reivindica polemicamente a própria ascendência hebraica: "São esses hebreus? Também eu. São israelitas? Também eu. São da estirpe da Abraão? Também eu" (2Cor 11,22). "Circunciso no oitavo dia, da estirpe de Israel, da tribo de Benjamin, hebreu dos hebreus, fariseu segundo a lei" (Fil 3,5-6). Assim, aos filipenses, percorre tais palavras, para ressaltar o novo estado no qual se encontra depois de ter sido assinalado por Cristo.

Os fariseus consideram simples o modo deles de viver, não fazendo nenhuma concessão à sua fragilidade. Seguem o que a sua doutrina escolheu e transmitem como bom, dando a máxima importância àqueles ordenamentos que consideram adaptados e ditados por eles. Tem respeito e consideração pelos seus anciãos e não ousam contradizer a proposta deles.

Sustentam que cada coisa é governada pelo Destino, mas não vetam à vontade humana de fazer o quanto está em seu poder, sendo prazeroso a Deus que se realizasse uma fusão: que o desejo do homem, com a sua virtude e o seu vício, fosse admitido na câmara do conselho do Destino. Crêem na imortalidade da alma, e

\footnotetext{
${ }^{2}$ Per il rapporto tra Paolo e l'ebraismo del I secolo(Cf. G. BoCCACCINI, Il Medio Giudaismo. Genova 1993; R. PENNA, "Un fariseo del Secolo I. Paolo di Tarso", Ricerche Storico-Bibliche (1999) 65-88; A. PITTA, "Paolo e il giudaismo farisaico", Ricerche Storico-Bibliche (1999) 89-108; C. TALBERT, "Paul, Judaism and the Revisionists", $C B Q 63$ (2001) 1-22; J.-N. ALETTI, "Où en sont les études sur S. Paul? Enjeux et propositions", Recherche de Science Religieuse 90/3 (2002) 339-343.
} 
que sob a terra existam recompensas e punições para os homens: a virtude ou vício; eterno castigo é a sorte das almas cativas, enquanto as almas boas recebem um fácil acesso para uma nova vida. Por causa destes (ensinamentos) têm um real e extremamente considerável influxo junto ao povo; e todas as orações e os sagrados ritos do culto divino são seguidos conforme suas disposições. A prática dos seus altíssimos ideais seja no mundo do viver, seja no dos pensamentos, é o eminente tributo que os habitantes das cidades pagam à excelência dos Fariseus ${ }^{3}$.

$\mathrm{Na}$ Carta aos Romanos, há uma lúcida consciência teológica de pertencer por nascimento ao povo chamado por Deus para um plano de salvação a favor de toda a humanidade: "Desejo ser eu mesmo maldito por Deus, separado por Cristo para os meus irmãos da minha estirpe que são israelitas e que possuem a adoção de filhos, a glória, a aliança, a lei, o culto, as promessas, os patriarcas e dos quais nasceu Cristo segundo a carne" ( $\mathrm{Rm} 9,3-5)$. Em um momento surge o orgulho separatista "por natureza sou hebreu e não como os gentios pecadores" (Gal 2,15).

Enquanto sentindo-se radicalmente convertido a Cristo, Paulo vive em um clima espiritual hebraico: quando fixa data de término de tempo, o faz em termos do calendário hebraico (cfr. 1 Cor 16,8); duas vezes os Atos o apresenta empenhado no voto de nazirato (cfr. At 18,18; 21,17 26). A Bíblia é o seu livro (isto é, a LXX), que ele usa e trata à maneira dos rabinos, seguindo-lhes no método de leitura e interpretação ${ }^{4}$.

Os Atos contêm a notícia do seu crescimento em Jerusalém e da sua formação "na escola de Gamaliel" (At 22,3) em conformidade às mais rígidas normas da lei dos pais. Ele deve à tradição hebraica também ter aprendido uma profissão, por motivos éticos de ordem utilitarista, o qual, no caso de Paulo, era aquele de 'fabricante de tendas' (termo genérico que permite diversas interpretações): tecelão de pele de cabra de vários tipos, utilizada para fazer tecidos ásperos para diversos usos, como o cilício (assim chamado porque procedente da Cilícia, de onde vinha

\footnotetext{
${ }^{3}$ G. FlaVIO, Antichità giudaiche, Libro XVIII, 12-15.

${ }^{4}$ Per esempio midrash (cfr. 1Cor 10,1-10) e gezerah shawah (cfr. Gal 3,10-14; Rom 4,3-8; 9,25-28). Il primo descrive ogni tipo di ricerca sulla Scrittura, mentre il secondo è una delle regole d'interpretazione per due passi della Scrittura che hanno uno o più termini in comune.

152 - Universidade Católica de Pernambuco
} 
confeccionado) ou trabalhador de peles e couros, utilizados para fabricar tendas para vários objetivos 5 .

Este hebreu era de Tarso, uma "não obscura cidade da Cilícia" (At 21,39), como ele mesmo com prazer o definia. Tarso, sob o rio Cidno, estava naquele tempo, no apogeu do seu esplendor de cidade helenística e cosmopolita. Em suma, a identidade hebraica de Paulo constitui o húmus principal no qual germinam todos os seus insights sobre a figura, missão e sobre o reino de Jesus Messias ${ }^{6}$.

\subsection{O helenismo}

Todo o quadro da sua atividade se coloca em um ambiente cultural helenista". Tarso era uma das "pátrias" do estoicismo:

5 "Scholars have usually accepted the evidence of Acts that Paul's trade was tentmaking but have gone on to debate whether Paul's trade involved making tents from goats' hair, linen, or leather. The first two options are more likely if, as is usually assumed, Paul learned his trade at home while a boy, as I argued previously, for goats' hair, that is, cilicium, would connect his trade to his province of Cilicia, whereas linen would, too, since it was a principal commodity of his hometown of Tarsus. But this connection with Tarsus does not work for Aquila and Priscilla, Paul's fellow tentmakers, since Aquila at least was from Pontus (Acts 18:2), and it would not work for Paul either, if he learned his trade after leaving Tarsus, as I will argue below. There are still advocates of Paul's trade as involving weaving, but the view that Paul made tents from leather, not to mention other leather products, remains - correctly, in my view - the dominant one" (R.F. Hock, "The Problem of Paul's Social Class", in Paul's World, 10).

6 “... gli aspetti più importanti del pensiero dell'Apostolo che gli derivano dal suo background giudaico: il ricorso fondante alle Scritture e la loro interpretazione (con Adamo e Abramo quali personaggi più citati), l'enfasi sul monoteismo, la conferma di Israele come popolo eletto da Dio, il riferimento al culto ebraico, la prospettiva morale (soprattutto per ciò che riguarda l'idea di peccato e la lista dei vizi), l'insistenza sulla Legge e sulle opere, il concetto di giustizia (umana e divina), la credenza nel giudizio finale e universale di Dio in connessione con la risurrezione della carne" (F. BIANCHINI, "Alla ricerca dell'identità dell'apostolo Paolo", 50).

${ }^{7}$ Per il rapporto tra l'ebraismo e l'ellenismo nel I secolo vedi E. BICKERMAN, Der Gott der Makkabäer, Berlin 1937; English trans., The God of the Maccabees. Studies in the Origin and Meaning of the Maccabean Revolt, Leiden 1979; V. TCHERIKOVER, Hellenistic Civilization and the Jews, Philadelphia 1959, Peabody (MA) 1999; M. HENGEL, Judentum und Hellenismus, Tübingen 1968; English trans., Judaism and Hellenism. Studies of their Encounter in Palestine during the Early Hellenistic Period, I-II, Philadelphia 1974; J.J. Collins, Between Athens 


\section{Ágora Filosófica}

Paulo teve certamente a oportunidade de conhecer este tipo de pensamento e dele assimilou certos traços éticos, como o ideal de autossuficiência (Cf. Fil 4,11; 2 Cor 9,8; 1Tm 6,6); do domínio de si (Cf. 1Cor 7,9; 9,25; Gal 5,23); e alguns conceitos filosóficoreligiosos, como a transparência de Deus no mundo (Cf. Rm 1,1920).

O Tarsiota usa o grego com desenvoltura e de maneira pessoal; não lhe são estranhas nem as formas da diatribe, nem as figuras da retórica contemporânea ${ }^{8}$, e se revela linguisticamente criativo. Basta pensar nos verbos formados com uma ou mais preposições (cfr. $\operatorname{Rm} 5,20$ : 8,26; 2 Cor 7,4), entre os quais, são típicos os compostos com su,n (= con) para indicar a simbiose com colaboradores e amigos na comunicação vital com Cristo na morte, na ressurreição e na glória (cfr. Rm 6,4ss; 8,17, Gal 2,19; Fil 3,10; Ef 2,6; Col 2,12; 3,1ss). Não são raros os casos nos quais os vocábulos em uso na cultura grega contemporânea, vêm escrito sob a sua pena para exprimir conteúdos e significados novos, conforme o seu pensamento teológico.

Basta pensar na dilatação e transformação semântica por ele empregadas aos termos chave: como carne (sarx) e espírito (pneuma); pecado (hamartia) e salvação (soteria); amor (agape); justiça (dikaiosyne); liberdade (eleutheria) e servidão (douleia). Em particular, o seu pensamento apresenta a situação existencial e cultural com a qual está em contato, ao ponto que se pode falar para ele de uma verdadeira e própria "inculturação" da fé em contextos diversos daquele hebraico-hierosolimitano no qual nasceu.

As duas cartas aos Coríntios e aquelas aos Efésios e aos

and Jerusalem. Jewish Identity in the Hellenistic Diaspora, Grand Rapids 1985, $2000^{2}$; L.I. LEVINE, Judaism and Hellenism in Antiquity. Conflict or Confluence? Seattle 1998.

8 “Anzitutto già a una lettura superficiale le sue epistole mostrano una pluralità di elementi retorici, mentre, a un'analisi più approfondita, la conoscenza della retorica e dei suoi procedimenti si rivela essenziale per comprendere il modo di argomentare dell'Apostolo e la coerenza dei suoi testi. Inoltre è necessario considerare che la cultura ellenistica del I secolo era profondamente intrisa di retorica [...] In definitiva, per gli interpreti di Paolo si tratta dunque di prendere le mosse nei loro studi dal cuore stesso di quella civiltà, per arrivare, successivamente, a comprendere che tipo di influsso esercitarono sull'Apostolo tutti i diversi elementi presenti in tale contesto culturale" (F. BIANCHINI, "Alla ricerca dell'identità dell'apostolo Paolo", 48).

154 • Universidade Católica dePernambuco 
Colossenses oferecem testemunho de maneira apropriada e convincente.

\subsection{A romanidade}

O apóstolo se auto apresenta em todas as cartas com o nome latino de Paulo, que trazia, quase certamente, desde o nascimento com aquele nome de Saulo. Os seus pais, com o nome de Saulo, faziam recordação do primeiro rei da tribo de Benjamin. Percebe-se que na Cristofania de Damasco, uma voz misteriosa o chama à maneira hebraica: "Saulo, Saulo" (At 9,4). Aos seus olhos, a autoridade do império responde à uma disposição divina: está " $a$ serviço de Deus para o teu bem" (Rm 13,4), por isso merece respeito e obediência "por motivo de consciência" ( $\mathrm{Rm} 13,5)$. Segundo o autor dos Atos dos Apóstolos, ele é confrontado serenamente e abertamente com pró-cônsules e procuradores romanos em Chipre, Corinto, Cesaréia, e reivindicou (não uma vez somente), as garantias jurídicas devido a isso, em força do direito de cidadania romana que possuía por nascimento (Cf. At 22,28).

Nos seus programas missionários, Roma figura como o vértice, o centro e a base de uma extensa evangelização, que deveria conduzi-lo até a Espanha (Cf. Rm 15,22-24), depois de tanto empenho que fizera na área oriental do Mediterrâneo. Não estamos em condições de estabelecer com segurança, se o grande sonho se tornou realidade - pelo menos nos termos por ele usado como acima citado - mas é muito provável. Em suma, a cidadania romana de Paulo, certamente sustentou e reforçou o universalismo da sua teologia e da sua missão apostólica? .

9 “... dobbiamo notare che negli studi recenti, di spiccata indole filosofica, si è andati a sottolineare l'universalismo dell'Apostolo, a fronte di ogni particolarismo di ordine etnico e religioso, facendo derivare questo elemento dalla sua cultura greco-ellenistica. Questo aspetto del pensiero di Paolo è, a nostro avviso, di grande rilevanza e costituisce la novità portata dalla sua figura all'interno della Chiesa primitiva. Egli non solo ha affermato come 'in Cristo' non siano più dirimenti le differenze etniche, sessuali, sociali (Gal 3,28) e come il vangelo sia offerto, senza alcuna distinzione, per la salvezza di ogni uomo, chiamato soltanto a credere in Cristo (Rm 1,16-17), ma ha effettivamente vissuto la sua missione con un respiro e un raggio di azione universali. In effetti, è proprio questa l'apertura nei confronti dei diversi popoli che l'impero romano evoca e prospetta, grazie alla rilevante estensione del suo dominio, ed è, secondo 


\subsection{Três mundos e três culturas}

Diante desta diversidade de planos culturais, como podemos definir a identidade do Apóstolo? Na sua pessoa e na sua obra se entrecruzam três mundos e três culturas: judaísmo, helenismo e romanidade. Paulo participa destes três níveis culturais fundamentais ao mesmo tempo. Sendo um homem cosmopolita e "globalizado"10, o Apóstolo dos gentios encontrou a sua razão de ser e a plena síntese dos seus planos culturais no anúncio incansável do senhorio de Jesus Cristo.

\section{A dispositio típica das cartas paulinas: tese e probatio segundo o ensinamento de Aristóteles}

Depois deste olhar panorâmico, agora aprofundarei o ambiente helenístico de Paulo, colocando em relevo, sobretudo seu domínio da língua grega e da retórica clássica. A arte de persuadir um público, seja em modo oral, seja em modo escrito, foi muito apreciado e prestigiado pelos antigos gregos e romanos. A figura do Apóstolo dos gentios nos oferece um belo exemplo do homem de cultura, que habilmente se comunicava com o seu público.

\subsection{A retórica grega e a Retórica de Aristóteles}

O termo genérico grego: téchnē rhētorikè, da época de Platão e depois o termo técnico: rhētorikéé, em latino: ars oratoria, ars dicendi (retórica como experiência adquirida) ou eloquentia (como habilidade). No coração do patrimônio cultural grecoromano se encontra - com a filosofia - a arte da retórica. ${ }^{11}$ Nenhuma outra civilização fora da Europa alcançou o mesmo nível

noi, penetrata come tale nella mentalità del cittadino di Tarso, il quale, una volta divenuto credente in Cristo, ha pienamente messo a frutto tale portato della sua cultura romana a esclusivo vantaggio dell'annuncio universale del vangelo (cf. Rm 15,17-21)" (F. BIANCHINI, "Alla ricerca dell'identità dell'apostolo Paolo", 52-53).

${ }^{10}$ Vedi G. BiguZZI, Paolo, comunicatore. Tra interculturalità e globalizzazione, Milano 1999.

${ }^{11}$ Vedi C. WALDE, "Rhetoric", Brill's New Pauly, XII, 530-536.

156 • Universidade Católica dePernambuco 
de elaboração e de sistematização nesta matéria. Para os gregos e os romanos, a retórica constituía uma verdadeira "metalíngua" que se refletia em várias formas na prática da vida quotidiana, sobretudo na política (por exemplo a polis grega, a república romana etc.). Como técnica, se pode definir a retórica como a arte da persuasão, isto é, o conjunto de regras e de prescrições utilizadas para persuadir o público seja em modo oral, seja em modo escrito.

Nesta relação, não tenho a intenção de fazer um resumo da história da retórica na Grécia clássica (as grandes figuras como Corace e Tisia de Siracusa, Górgias, Isócrates, Platão etc.), mas, propriamente, enfatizar a figura de Aristóteles e a sua obra magistral a Retórica. Aristóteles explica que um discurso deve ter uma dupla composição, isto é, possuir uma proposição (propositio) e as provas (argomentatio ou probatio).

Duas são as partes do discurso: é de fato, necessário primeiro expor o argumento em torno ao qual se fala e então demonstrá-lo. Por isso, é impossível que aquele o expõe não demonstre, e quem o demonstre não o tenha exposto. De fato, quem demonstra, demonstra qualquer coisa, e quem coloca a premissa de qualquer coisa, o faz para depois a demonstrar. Destas duas partes, a primeira é a próthesis (proposição, apresentação da tese para demonstrar), e a segunda é a pístis (argumentação, prova). (Retorica 1414a).

Os elementos fixos e obrigatórios (sempre presentes) de um discurso são três: o exórdio, a argumentação e a conclusão; enquanto os elementos móveis e não indispensáveis (nem sempre presentes) são quatro: a narração, a proposição, a divisão e a digressão. As argumentações paulinas parecem seguir a seu modo, este tipo de disposição ${ }^{12}$.

\subsection{A retórica grega antiga e o significado da terminologia hoje}

A disposição ordenada (lat. dispositio; ing. "arrangement"; fr. "disposition", "ordre", "ordonnancement") de uma oração ou de uma carta antiga, se insere na estrutura de qualquer discurso, seja esse deliberativo, judicial ou demonstrativo. ${ }^{13} \mathrm{~A}$ disposição coloca

\footnotetext{
${ }^{12}$ Vedi J.-N. AletTI - al., Lessico ragionato dell'esegesi biblica, 92-93.

${ }^{13}$ Diamo qui di seguito la tripartizione dell'oratoria classica greca ereditata anche
} 
em ordem os argumentos que se desejam tratar. Hoje, alguns estudiosos reconhecem os mesmos elementos nas cartas antigas de Platão a Sêneca. Isso também ocorre com as cartas de São Paulo. Mesmo os autores contemporâneos preferem usar a terminologia latina para identificar estas repartições, com a finalidade de evitar equívocos. Além de acrescentarem outros elementos retóricos dos manuais clássicos para explicar mais exaustivamente o esquema constitutivo das cartas paulinas. Eis uma lista de máximas, na qual estão elencadas e sinteticamente explicadas, as partes segundo as quais, genericamente pode ser subdividida uma carta paulina. ${ }^{14}$

\section{a) exordium}

O exórdio é uma breve introdução do discurso. Deve conferir o tom, estabelecer um contato com os destinatários e anunciar o argumento e os temas principais. Compreende dois momentos: 1) a captatio benevolentiae e 2) a propositio (e se há, a partitio).

\section{b) captatio benevolentiae}

Figura retórica que retorna ao topos, e com o qual o autor tenta suscitar uma atitude benévola da parte do destinatário. Nas composições literárias se encontra geralmente no início do exórdio.

\section{c) propositio}

Enunciação geral elaborada e complexa de uma seção da matéria (thema) que o autor está tratando ou se prepara para tratar; enunciação de especial importância, situada no coração da carta e

nel mondo latino: 1) dikaniko.n ge,noj - genus iudiciale - genere giudiziario (o forense); 2) sumbouleutiko.n ge,noj — genus deliberativum — genere deliberativo; 3) evpideiktiko.n ge,noj — genus demonstrativum - genere epidittico (o dimostrativo). Cfr. H. LAUSBERG, Elementi di retorica, $\S 22$.

${ }^{14}$ Per brevi definizioni di queste voci, si veda J.-N. ALETTI, La lettera ai Romani e la giustizia di Dio, Roma 1997, 266-269; ID., Comment Dieu est-il juste? Clefs pour interpréter l'épître aux Romains, Paris 1991, 259-265; J. MURPHYO'Connor, Paul the Letter-Writer. His World, His Options, His Skills, Collegeville, Minnesota 1995, 71-77.

158 - Universidade Católica dePernambuco 
contendo a demonstração dos argumentos dos quais ele deseja obter a aprovação. Para ser considerado uma propositio (segundo momento do exórdio), o enunciado deve ser acompanhado de um desenvolvimento amplo, que possa justificá-la. Segundo alguns biblistas (entre os quais Aletti e Pita), a propositio é a tese principal da carta.

\section{d) partitio}

A partitio anuncia o plano que o autor está para seguir; inclui de qualquer modo os pontos essenciais ou partes principais da demonstração. Se o conteúdo é organizado de modo mais detalhado, à propositio (segundo momento do exórdio) segue uma partitio.

\section{e) narratio}

A narração é o exposto inicial que apresenta os fatos e explica o background da situação do remetente e dos destinatários. A narratio deve ser breve e concisa, porque tende a preparar indiretamente a confirmatio/probatio/argumentatio.

\section{f) probatio, argumentatio, confirmatio}

A argumentação representa o coração do discurso persuasivo. Nesta, o autor desenvolve os argumentos que comprovam a sua tese e a confirma do seu ponto de vista. Se trata do corpo principal do desenvolvimento. Para exemplificar, neste texto, não insisto sobre a diferença entre estes termos porque todos designam o corpo da argumentação.

\section{g) subpropositio}

Breve enunciação relativa ao argumento tratado no interior de uma das seções e na qual a carta está subdividida; a subpropositio representa então a enunciação de uma microunidade. 
h) ratio $^{15}$

É a razão que segue imediatamente a propositio e apresenta uma primeira e brevíssima justificação.

\section{i) correctio}

É uma espécie de esclarecimento semântico. Há dois tipos principais: a contraposição 'não $p$, mas $q$ ' (com as variantes estilísticas: ' $q$, não / antes que $q$ ' etc.) e melhoramento: ' $p$ ou melhor / para melhor dizer etc. $q$.'

\section{j) transitio}

A atenção dos limites estruturais vem por meio da inserção de uma transitio, isto é, uma transição, passagem (atua como fechamento).

\section{1) digressio}

A digressão é uma parte do discurso com funções diversas (por ex.: faz repousar os destinatários ou ampliar a sua liberdade de juízo). Recusada por Aristóteles, esta parte facultativa foi retomada pelos romanos. O vocábulo não significa algo totalmente pejorativo.

m) peroratio

Peroração do discurso, isto é, epílogo ou conclusão, que recapitulando a argumentação, atua como fechamento. Compreende dois níveis: 1) posita in rebus nível das "ações" e das "vontades", e 2) posita in affectibus ou nível dos "sentimentos".

Em suma, a dispositio significa a colocação das grandes partes do discurso. Para uma clara apresentação da composição da dispositio, vejamos R. Barthes, La retorica antica, 90-91:

A dispositio parte de uma dicotomia que já existia. Em outros

15 Al plurale "rationes" = prove.

160 • Universidade Católica dePernambuco 
termos, aquela da Inventio: animos impellere (comover-se) / rem docēre (informar, convencer). $\mathrm{O}$ primeiro termo (o apelo aos sentimentos) cobre o exórdio e o epílogo, isto é, as duas partes extremas do discurso. $\mathrm{O}$ segundo termo (o apelo ao fato, à razão) cobre a narratio (relação dos fatos) e a confirmatio (verificação das provas ou vias de persuasão), isto é, as duas partes medianas do discurso. A ordem sintagmática não segue então a ordem paradigmática e assim, temos uma estrutura em quiasmo: duas seções "passionais" enquadram um bloco demonstrativo:

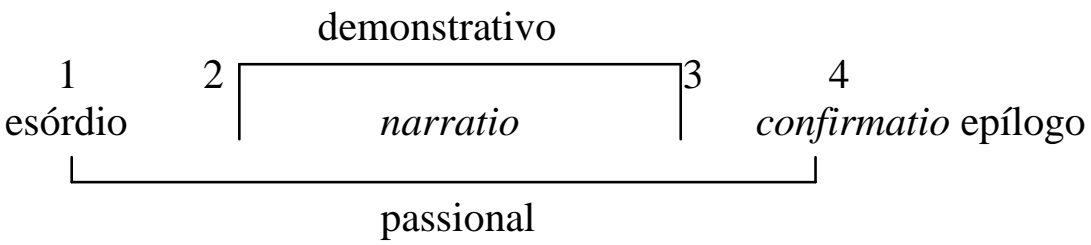

\subsection{A Carta aos Romanos como exemplo da dispositio aristotélica}

Se a composição da Carta aos Romanos fez surgir muitas dificuldades no passado, hoje não é mais assim: a exceção do cap. $5^{16}$, o consenso entre os biblistas é quase universal ${ }^{17}$. Consideramos diversos critérios de composição, indicativos do desenlace da carta. Segundo o biblista francês J.-N. Aletti, professor emérito do Instituto Bíblico de Roma, a dispositio retórica da Carta aos Romanos seria a seguinte. ${ }^{18}$

\footnotetext{
${ }^{16}$ Quattro sono le posizioni degli studiosi sulla funzione di Rm 5: 1) Rm 5,1-21 appartiene al blocco formato da $\mathrm{Rm} 1,18-5,21$; 2) $\mathrm{Rm} 5,1-21$ dà 1'avvio una nuova sezione, che finirebbe — secondo i più — a Rm 8,39; 3) Rm 5,1-11 funge da conclusione per la parte 1,18-5,11 e Rm 5,12 segna l'inizio di una nuova sezione; 4) Rm 5 è un'unità isolata. Cfr. J.A. FITZMYER, Romans, 96-97 e J.-N. AletTI, Comment Dieu est-il juste?, 38-49.

17 Per uno studio più approfondito di questo problema, vedi J.-N. ALETTI, Comment Dieu est-il juste?, 38-49.

${ }^{18}$ Cfr. J.-N. AletTi, "Romans", IBC, 1554; ID., La Lettera ai Romani, 9. Cfr. S. LYONNET, "Note sur le plan de l'épître aux Romains," RSR 39 (1951) 301-316; R. SCROGGS, "Paul as Rhetorician. Two Homilies in Romans 1-11", in R.G. HAMERTON-Kelly, ed., Jews, Greeks and Christians. Religious Cultures in Late Antiquity, Leiden 1976, 271-298; J.A. FITZMYER, Romans, 96-102. “...per non costringere e mutilare i testi biblici su di un ideale 'letto di Procuste' è necessario
} 
praescriptum $1,1-7$

exordium 1,8-17 apêndice de propositio generalis (vv. 16-17)

probatio $\quad 1,18-11,36$
A. $1,18-4,25$
B. $5-8$
C. $9-11$

exortações

$12,1-15,13$

peroratio

$15,14-21$

notícias

saudação final 16,1-27

A propositio generalis (tese principal) de Romanos enuncia todos os grandes temas teológicos que o Apóstolo das gentes deseja desenvolver na sua epístola aos crentes de Roma: "Eu de fato, não me envergonho do Evangelho, porque é força de Deus para a salvação de todo o que crê, do Judeu, primeiro, como do Grego. Nisto de fato, se revela a justiça de Deus, da fé para a fé, como está escrito: 'o justo pela fé viverá” (Rm 1,16-17).

A probatio (prova ou argumentações, isto é, a parte doutrinal ou dogmática da carta) demonstra alguns paralelos lexicográficos e temáticos que se podem subdividir em três argumentos: 1) nos caps.1-4 está ilustrada a justificação já obtida

procedere dal testo, con una sua composizione, struttura o disposizione, e semmai ad un relativo genere retorico. Infine è necessario tener presente che i modelli compositivi della manualistica classica non venivano seguiti sic et simpliciter da coloro che intendevano persuadere gli ascoltatori: si era pienamente liberi nel disporre la comunicazione a proprio piacimento. Questo vale anche per gli autori del NT, liberi di fronte ai canoni della retorica, della narrazione e dell'epistolografia, per cui si deve con pertinenza parlare di 'retorica contro la retorica', senza tuttavia cadere nell'assunto che non esista una retorica neotestamentaria. Anche gli autori del NT dimostrano un proprio modo di fare retorica più impronta al kérigma che ai canoni estetici del linguaggio. [...] Dunque il percorso contrario del rhetorical criticism genera la nuova denominazione della 'retorica letteraria', in cui per 'letteraria' s'intende l'esigenza di conferire priorità al testo biblico, con le sue micro e macrounità" (A. PITTA, "Retorica biblica", 1363). 
em Cristo somente pela fé n'Ele ${ }^{19} ; 2$ ) os caps. 5-8 tratam das consequências de tal justificação pela vida presente e futura dos cristãos; 3) nos caps. 9-11 se examina a questão da salvação de Israel $^{20}$. A causa, seja da argumentação escriturística, seja da temática teológica que a une, 1,18-4,25 e 9-11, constituem o paralelismo principal das asas da probatio, a qual tem uma estrutura concêntrica.
A. $=1,18-4,25$
Hebreu e grego justificados por meio da fé
B. $=5-8$ vida nova e esperança dos justificados
$\mathrm{A}^{\prime} .=9-11$
Israel e os gentios: a salvação de Israel

Em toda a parte doutrinal, se instaura uma tensão entre o ‘já' de agora e o 'ainda não'. Os batizados são atualmente já justificados e redimidos em Cristo, mas ainda não estão vivendo a plenitude da salvação que se realizará somente no momento da parusia gloriosa do Senhor ressuscitado ${ }^{21}$.

19 "The attention to the eminent rhetorical arrangement in Romans has thus allowed overcoming a major difficulty, that of the connection between the main propositio of 1:16f. and the development that is immediately supposed to justify it, 1:18-4:25. We have seen how Paul arrives at showing, without any incoherence and in stages, (1:18-3:20 and 3:21-4:25) that divine justice reaches all humans in the same way, that is to say, by faith without the works of the Law. This then is the main quality of divine justice maintained by Paul and which he expresses as justification by faith alone: beyond the constancy of His judgment, the firmness with which He executes retribution, God is just because He treats all His subjects without making exceptions or giving any privileges. Indeed, the way in which Paul begins his reasoning in 1:18-3:20 can today raise questionsbased on the falseness of mankind, is it necessary to highlight the truth of God and to proclaim the Gospel of gracious justification?-but we have seen that it is the imperatives of rhetoric that determine this point of departure" (J.-N. ALETTI, God's Justice in Romans, 90-91). Vedi anche F. BIANCHINI, L'analisi retorica delle lettere paoline, 45-54.

${ }^{20}$ Vedi B. BYRNE, Galatians and Romans, 56-58.

${ }^{21}$ La giustificazione per Paolo è una realtà ben radicata nel presente, mentre la salvezza è una realtà futura (cfr. Rm 5,1.10). In $\mathrm{Rm} 5,1$ si dimostra che la giustificazione è una realtà presente: Dikaiwqe,ntej ou=n evk pi,stewj eivrh,nhn e;comen pro.j to.n qeo.n dia. tou/ kuri,ou h`mw/n VIhsou/ Cristou/) In Rm 5,10 si vede che la salvezza è una realtà futura: pollw// ma/llon katallage,ntej swqhso,meqa evn th/| zwh/| auvtou/. Questo vale anche per Israele, perché Dio rimane sempre fedele al suo popolo eletto e lo salverà secondo il suo progetto di amore: kai. ou[twj pa/j VIsrah.l swqh,setai (Rm 11,26). 


\section{Conclusão}

Aristóteles e o Apóstolo Paulo, duas grandes personalidades do mundo antigo, à primeira vista, parecem pertencer a dois mundos distintos, dois Weltanschauung completamente independentes. Espero que a minha conferência vos convença do oposto: a riqueza cultural do Apóstolo das gentes, graças aos seus três mundos culturais, o coloca no coração do helenismo do primeiro século depois de Cristo. O nosso exame da disposição típica das cartas paulinas: tese e probatio segundo o ensinamento de Aristóteles é a prova mais convincente. Depois, a nossa breve consideração da Carta aos Romanos, como exemplo típico da dispositio aristotélica, nos serviu para aprofundar esta preciosíssima temática. Aristóteles e Paulo, por séculos, duas colunas notáveis da civilização ocidental, encontraram terra comum na retórica grega (clássica e depois helenística) pela articulação detalhada do pensamento profundo e riquíssimo deles.

\section{Referências}

Aletti, J.-N., «La présence d'un modèle rhétorique en Romains. Son rôle et son importance », Bib. 71 (1990) 1-24.

"La dispositio rhétorique dans les épîtres pauliennes. Propositions de méthode", NTS 38 (1992) 385-401.

- "Paul et la rhétorique. État de la question et proposition", in J. Schlosser, ed., Paul de Tarse, LeDiv 165, Paris 1996, 27-50.

- "La rhétorique paulinienne : construction et communication d'une pensée", in A. Dettwiler - J.-D. Kaestli - D. Marguerat, ed., Paul, une théologie en construction, MoBi 51, Genève 2004, 47-66.

Aletti, J.-N. - al., Vocabulaire raisonné de l'exégèse biblique. Les mots, les approches, les auteurs, Paris 2005 ; trad. italiana, Lessico ragionato dell'esegesi biblica. Le parole, gli approcci, gli autori, Brescia 2006; trad. española, Vocabulario razonado de la exégesis bíblica. Los términos, las aproximaciones, los autores, Instrumentos para el estudio de la Biblia 15, Estella 2007. SL 10 L 10; SL 10 E 136; SL 10 E 139 Anderson, R.D., Ancient Rhetorical Theory and Paul, Leuven 1999. Aune, D.E., The New Testament and Its Literary Environment, Philadelphia 1987.

Forms and Genres, Atlanta 1988.

-, The Westminster Dictionary of the New Testament and Early 164 • Universidade Católica dePernambuco 
Christian Rhetoric, Louisville - London, 2003.

Barthes, R., L'ancienne rhétorique, Paris 1970; trad. italiana, La retorica antica. Alle origini del linguaggio letterario e delle tecniche di comunicazione, Milano 1994.

Betz, H.D., "The Problem of Rhetoric and Theology According to the Apostle Paul", in A. Vanhoye, ed., L'Apôtre Paul. Personnalité, style et conception du ministère, BEThL 73, Leuven 1986, 16-48.

Botha, P.J.J., "The Verbal Art of the Pauline Letters. Rhetoric, Performance and Presence", in S.E. Porter, ed., Rhetoric and the New Testament. Essays from the 1992 Heidelberg Conference, JSNTSS 90, Sheffield 1993, 409-428.

Classen, C.J., "Paulus und die Antike Rhetorik", ZNW 82 (1991) 1-33.

_- "St. Paul's Epistles and Ancient Greek and Roman Rhetoric", in

S.E. Porter, ed., Rhetoric and the New Testament. Essays from the 1992 Heidelberg Conference, JSNTSS 90, Sheffield 1993, 265-291.

Daube, D., "Rabbinic Methods of Interpretation and Hellenistic Rhetoric", HUCA 22 (1949) 234-264.

Dormeyer, D., "The Hellenistic Letter-Formula and the Pauline LetterScheme", in S.E. Porter, The Pauline Canon, Pauline Studies (Past) 1, Leiden - Boston, 2004, 59-93.

Doty, W., "Classification of Epistolary Literature", CBQ 31 (1969) 183199. 90 (1971) 91-97.

- Letters in Primitive Christianity, Philadelphia 1973, $1977^{2}$.

Exler, F.X.J., The Form of the Ancient Greek Letter. A Study in Greek Epistolography, Washington 1923, $1974^{2}$.

Kennedy, G.A., The Art of Persuasion in Greece, Princeton 1963.

, The Art of Persuasion in the Roman World (300 B.C.-AD300), Princeton 1972.

, Classical Rhetoric and its Christian and Secular Tradition from Ancient to Modern Times, Chapel Hill 1980.

- A New History of Classical Rhetoric. An Extensive Revision and Abridgment of The Art of Persuasion in Greece, The Art of Rhetoric in The Roman World, and Greek Rhetoric under Christian Emperors, with Additional Discussion of Late Latin Rhetoric, Princeton, 1994.

- New Testament Interpretation through Rhetorical Criticism, Chapel Hill 1984.

- Progymnasmata. Greek Textbooks of Prose Composition and Rhetoric, Leiden - Boston, 2003.

Kinneavy, J.L., Greek Rhetorical Origins of Christian Faith. An Inquiry, Oxford - New York, 1987.

Lambrecht, J., "Rhetorical Criticism and the New Testament", Bijdragen 
50 (1989) 239-253.

Lanci, J.R., Rhetorical and Archaeological Approaches to Pauline Imagery, New York 1997.

Lausberg, H., Handbuch der literarischen Rhetorik. Eine Grundlegung der Literaturwissenschaft, I-II, München 1960; English trans., Handbook of Literary Rhetoric, Leiden 1998; trad. española, Manual de retórica literaria, I-III, Madrid 1966. SL 800 R 7; SL 800 R 4/1-2; SL 800 R 3/13

- Elemente der literarischen Rhetorik. Eine Einführung für Studierende del klassischen, romanischen, englischen und deutschen Philologie, Ismaning 1963, 1990 ${ }^{10}$; trad. española, Elementos de retórica literaria, Madrid 1993; trad. italiana, Elementi di retorica, Bologna 1969. SL 800 R 11; SL 800 R 12; SL 800 R 5

Levison, J.R., "Did the Spirit Inspire Rhetoric? An Exploration of George Kennedy's Definition of Early Christian Rhetoric", in D.F. Watson, ed., Persuasive Artistry. Studies in New Testament Rhetoric in Honor of George A. Kennedy, Fs. G.A. Kennedy, Sheffield 1991, 25-40.

Mack, B.L., Rhetoric and the New Testament, Minneapolis 1990.

Malherbe, A.J., Ancient Epistolary Theorists, SBLSBS 19, Atlanta 1988.

Marchese, A., Dizionario di retorica e di stilistica. Arte e artificio nell'uso delle parole retorica, stilistica, metrica, teoria della letteratura, Milano 1991. SL 800 R 8

Martin, J., Antike Rhetorik. Technik und Methode, München 1974.

Mortara Garavelli, B., Manuale di retorica, Milano 1988, 19937. SL 800 R 6

Martin, J., Antike Rhetorik, München 1974.

Murphy-O'Connor, J., Paul the Letter-Writer. His World, His Options, His Skills, Collegeville 1995; French trans., Paul et l'art épistolaire. Contexte et structure littéraires, Paris 1994.

Patton, J. and V. Robbins, "Rhetoric and Biblical Criticism", Quarterly Journal of Speech 66 327-336.

Perelman, Ch., Realm of Rhetoric, Notre Dame 1982.

Perelman, Ch. - Olbrechts-Tyteca, L., Traité de l'argumentation. La nouvelle rhétorique, Paris 1958; trad. italiana, Trattato dell'argomentazione. La nuova retorica, Torino 1989.

Pennacini, A., "Retorica Classica", in Dizionario di Omiletica, ed. M. Sodi - A.M. Triacca, Torino 1998, 1355-1361.

Pitta, A., "Come si persuade un uomo? Analisi retorico-letteraria della lettera a Filemone", in C. Marcheselli-Casale, ed., Oltre il racconto. Esegesi ed ermeneutica: alla ricerca del senso, Napoli 1994, 93-108.

- Sinossi paolina, Cinisello Balsamo 1994.

, "Così 'inesperto nell'arte retorica'? (cf. 2Cor 11,6). Retorica e messaggio paolino", in La parola di Dio cresceva (At 12,24), Fs. C.M.

166 - Universidade Católica de Pernambuco 
Martini, Supplementi alla Rivista Biblica 33, Bologna 1998, 411-435. "Retorica Biblica", in Dizionario di Omiletica, ed. M. Sodi A.M. Triacca, Torino 1998, 1361-1365.

Porter, S.E., "The Theoretical Justification for Application of Rhetorical Categories to Pauline Epistolary Literature", in Id, ed., Rhetoric and the New Testament. Essays from the 1992 Heidelberg Conference, JSNTSS 90, Sheffield 1993, 100-121.

—, ed., Handbook of Classical Rhetoric in the Hellenistic Period 330 B.C. - A.D. 400, Leiden - New York - Köln 1997.

Probst, H., Paulus und der Brief. Die Rhetorik des Antiken Briefes als Form der Paulinischen Korintherkorrispondenz (1Kor 8-10), WUNT 45, Tübingen 1991.

Reed, J.T., "Using Ancient Rhetorical Categories to Interpret Paul's Letters. A Question of Genre", in S.E. Porter, ed., Rhetoric and the New Testament. Essays from the 1992 Heidelberg Conference, JSNTSS 90, Sheffield 1993, 292-324.

Richards, E., The Secretary in the Letters of Paul, WUNT 42, Tübingen 1991.

Schiappa, E., The Beginnings of Rhetorical Theory in Classical Greece, London 1999.

Sloan, T.O., ed., Encyclopedia of Rhetoric, Oxford 2001.

Stowers, S., Letter Writing in Greco-Roman Antiquity, Philadelphia 1986. Squarotti, G.B. - Gorrasi, G. - Meliga, W. - Molinaro, C., ed., Dizionario di retorica e stilistica. Metrica classica e moderna, figure e artifici retorica, generi, forme e strutture letterarie, concetti e fenomeni linguistici, elementi di grammatica, morfologia e sintassi, TEA, Torino 1995.

Thurén, L., "On Studying Ethical Argumentation and Persuasion in the New Testament", in S.E. Porter, ed., Rhetoric and the New Testament. Essays from the 1992 Heidelberg Conference, JSNTSS 90, Sheffield 1993, 464-478.

Watson, D.F., ed., Persuasive Artistry. Studies in New Testament Rhetoric in Honor of George A. Kennedy, Sheffield 1991.

—, "Rhetorical Criticism, New Testament", in Dictionary of Biblical Interpretation, ed. J. Hayes, Nashville 1999, 399-402.

Watson, D.F. - Hauser, J.A., Rhetorical Criticism of the Bible. A Comprehensive Bibliography with Notes on History and Method, Leiden - Boston 1994.

White, J.L., "Introductory Formulae in the Body of the Pauline Letter", JBL 90 (1971) 91-97.

, The Form and Function of the Body of the Greek Letter. A Study of the Letter-Body in the Non-Literary Papyri and in Paul the Apostle, SBLDS 2, Missoula MT 1972. 
—, "New Testament Epistolary Literature in the Framework of Ancient Epistolography", in ANWR, II25.2, Berlin - New York 1984, 1730-1756.

—, Light from Ancient Letters, Philadelphia 1986.

-, "Ancient Greek Letters", in D.E. Aune, ed., Greco-Roman Literature and the New Testament, Atlanta 1988, 85-105.

- "Apostolic Mission and Apostolic Message. Congruence in Paul's Epistolary Rhetoric, Structure and Imagery", in B.H. McLean, ed., Origins and Method. Toward a New Understanding of Judaism and Christianity, JSNTSS 86, Fs. J.C. Hurd, Sheffield 1993, 145-161.

Wuellner, W., "Greek Rhetoric and Pauline Argumentation", in Early Christian Literature and the Classical Intellectual Tradition, ed. W. Schoedel and R. Wilken, Paris 1979, 177-188.

- "Where is Rhetorical Criticism Taking Us?", CBQ 49 (1987) 448-463. 